\title{
Broadband DOA Estimation Based on Nested Arrays
}

\author{
Zhi-bo Shen, Chun-xi Dong, Yang-yang Dong, Guo-qing Zhao, and Long Huang \\ The Key Laboratory of Electronic Information Countermeasure and Simulation Technology, Ministry of Education, \\ Xidian University, Xian, Shaanxi 710071, China \\ Correspondence should be addressed to Chun-xi Dong; chxdong@mail.xidian.edu.cn
}

Received 21 October 2014; Accepted 24 March 2015

Academic Editor: Diego Masotti

Copyright (C) 2015 Zhi-bo Shen et al. This is an open access article distributed under the Creative Commons Attribution License, which permits unrestricted use, distribution, and reproduction in any medium, provided the original work is properly cited.

Direction of arrival (DOA) estimation is a crucial problem in electronic reconnaissance. A novel broadband DOA estimation method utilizing nested arrays is devised in this paper, which is capable of estimating the frequencies and DOAs of multiple narrowband signals in broadbands, even though they may have different carrier frequencies. The proposed method converts the DOA estimation of multiple signals with different frequencies into the spatial frequency estimation. Then, the DOAs and frequencies are pair matched by sparse recovery. It is possible to significantly increase the degrees of freedom (DOF) with the nested arrays and the number of sources can be more than that of sensor array. In addition, the method can achieve high estimation precision without the two-dimensional search process in frequency and angle domain. The validity of the proposed method is verified by theoretic analysis and simulation results.

\section{Introduction}

Direction of arrival (DOA) has been an active research area, playing an important role in electronic reconnaissance [1], as it is a crucial parameter for sorting and recognition of sources, directing jamming and passive location. Unlike radar receiver, there is no priori information of signals for the reconnaissance receiver. Therefore, the reconnaissance receiver tends to have large instantaneous bandwidth in order to cover a wide spectrum [2-4]. This leads to two problems in DOA estimation: one is that the array aperture must be increased to satisfy multiple signals of different frequencies within the reconnaissance band range and the other is that it must be possible to achieve the frequency and DOA estimation of multiple signals simultaneously in broadband. In [5], a nested array structure was proposed to increase the degrees of freedom by vectorizing the covariance matrix of the received signals among different sensors. Then, Pal and Vaidyanathan increase degrees of freedom of the coarray by extending the nesting strategy to multiple levels in [6], namely, the 2qthorder nested array, whose $2 q$ th-order difference coarray is proved to contain a uniform linear array with $O\left(N^{2 q}\right)$ sensors. It can be viewed as a virtual array with a wider aperture, which is capable of being utilized to improve the DOA estimation performance of the multiple signals with same frequency. However, when the frequencies of the signals are different and unknown, this technique cannot be applied directly. By exploiting the four-level nested array, a novel estimation approach is proposed in [7], which can be extended to wideband scenarios. However, for each subband signal, the vectorization is performed twice to construct a fourth order difference coarray and the spectrum peak search process is conducted in the whole spatial domain, which suffers from the complex computation. Signal subspace techniques such as MUSIC [8] need two-dimensional search process in frequency and angle domain, which leads to large amount of computation. In [9], an angle-frequency joint dictionary is established to achieve the frequency and DOA estimation by sparse recovery. The results suggest that sparse recovery becomes more difficult and complicated while the length of dictionary increases.

A broadband DOA estimation method is investigated in this paper. A nested array is used to extend the array aperture and the spatial frequency, which contains the information of both frequency and angle, is defined. In this way, the DOA estimation of multiple signals with different frequencies is converted into the spatial frequency estimation. Then, the received signals of sensor array are transformed into 


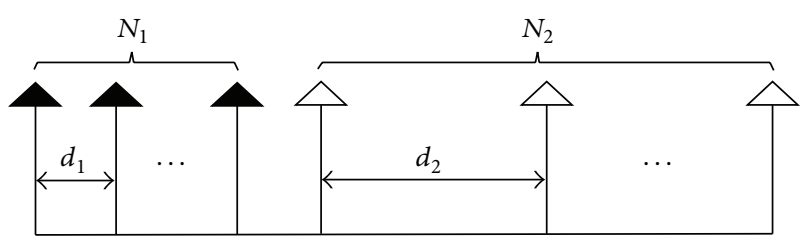

Figure 1: A 2-level nested array with $N_{1}$ sensors in the inner ULA and $N_{2}$ sensors in the outer ULA, with spacings $d_{1}$ and $d_{2}$, respectively.

frequency domain. By sparse recovery approach, each of the signals with different frequency can find its corresponding spatial frequency in the dictionary composed of spatial frequencies that have been estimated. Once the frequency and spatial frequency are pair matched, DOA estimation can be implemented simply by the definition of spatial frequency.

This paper is organized as follows. In Section 2, we introduce the nested array model. In Section 3, we propose the broadband DOA estimation method based on nested array and analyze the performance. Section 4 presents the simulation results, and conclusions are made in Section 5.

\section{The Nested Array Model}

A two-level nested array [10] with $N$ sensors is basically a concatenation of two uniform linear arrays (ULAs): inner and outer, where the inner ULA has $N_{1}$ sensors with spacing $d_{1}$ and the outer ULA has $N_{2}$ sensors with spacing $d_{2}=$ $\left(N_{1}+1\right) d_{1}$, as shown in Figure 1 . The resulting $N$ sensors are positioned at $S=\left\{n_{1} d_{1}, n_{1}=0,1, \ldots, N_{1}-1\right\} \cup\left\{n_{2} d_{2}-\right.$ $\left.d_{1}, n_{2}=1,2, \ldots N_{2}\right\}$, in which the first sensor is set to be the reference.

Assume that there are $K$ sources imping on the array from directions $\theta_{1}, \theta_{2}, \ldots, \theta_{K}$ with carrier frequency $f_{1}, f_{2}, \ldots, f_{K}$, respectively. The steering vector $\mathbf{a}\left(\theta_{i}, f_{i}\right)$ corresponding to the $i$ th source signal is expressed as

$$
\begin{gathered}
\mathbf{a}\left(\theta_{i}, f_{i}\right)=\left[1, e^{-j 2 \pi d_{1} f_{i} \sin \theta_{i} / c}, \ldots, e^{-j 2 \pi\left(N_{1}-1\right) d_{1} f_{i} \sin \theta_{i} / c},\right. \\
\left.e^{-j 2 \pi\left(d_{2}-d_{1}\right) f_{i} \sin \theta_{i} / c}, \ldots, e^{-j 2 \pi\left(N_{2} d_{2}-d_{1}\right) f_{i} \sin \theta_{i} / c}\right]^{\mathrm{T}},
\end{gathered}
$$

where $c$ is the velocity of wave propagation. The data vector received at the nested array is expressed as

$$
\mathbf{x}(t)=\sum_{i=1}^{K} \mathbf{a}\left(\theta_{i}, f_{i}\right) s_{i}(t)+\mathbf{n}(t)=\mathbf{A s}(t)+\mathbf{n}(t),
$$

where

$$
\begin{aligned}
\mathbf{A} & =\left[\begin{array}{llll}
\mathbf{a}\left(\theta_{1}, f_{1}\right) & \mathbf{a}\left(\theta_{2}, f_{2}\right) & \cdots & \mathbf{a}\left(\theta_{K}, f_{K}\right)
\end{array}\right], \\
\mathbf{s}(t) & =\left[\begin{array}{llll}
s_{1}(t) & s_{2}(t) & \cdots & s_{K}(t)
\end{array}\right]^{\mathrm{T}},
\end{aligned}
$$

where $\mathbf{A}$ denotes the array manifold matrix and $\mathbf{s}(t)$ denotes the source signal vector. $\mathbf{n}(t)$ is the white noise, which is uncorrelated from the sources.

\section{The Nested Array Model}

3.1. Spatial Frequency Estimation. From (1)-(4), it is obvious that there is two-dimensional information in broadband DOA estimation, namely, frequency and angle. Therefore, we attempt to estimate spatial frequency instead of frequency and angle, and the spatial frequency $\Omega$ is defined as

$$
\Omega=\frac{f \sin \theta}{c} .
$$

Combining (1) and (3), we obtain

$$
\mathbf{A}=\left[\begin{array}{llll}
\mathbf{a}\left(\Omega_{1}\right) & \mathbf{a}\left(\Omega_{2}\right) & \cdots & \mathbf{a}\left(\Omega_{K}\right)
\end{array}\right],
$$

where

$$
\begin{aligned}
& \mathbf{a}\left(\Omega_{i}\right)=\left[1, e^{-j 2 \pi d_{1} \Omega_{i}}, \ldots, e^{-j 2 \pi\left(N_{1}-1\right) d_{1} \Omega_{i}}, e^{-j 2 \pi\left(d_{2}-d_{1}\right) \Omega_{i}},\right. \\
& \left.\ldots, e^{-j 2 \pi\left(N_{2} d_{2}-d_{1}\right) \Omega_{i}}\right]^{\mathrm{T}} .
\end{aligned}
$$

Here, we assume that the sources are mutually uncorrelated and each source signal $s_{i}(t)$ is wide-sense quasistationary [11] with frame length $L$; that is,

$$
\begin{aligned}
E\left\{\left|s_{i}(t)\right|^{2}\right\} & =\sigma_{m i}^{2}, \\
& \forall t \in[(m-1) L, m L-1], m=1,2, \ldots, M .
\end{aligned}
$$

Then, the autocorrelation matrix of the $m$ th frame received signal is

$$
\begin{aligned}
& \mathbf{R}_{m}=E\left[\mathbf{x}(t) \mathbf{x}^{H}(t)\right]=\mathbf{A D}_{m} \mathbf{A}^{H}+\sigma_{n}^{2} \mathbf{I}, \\
& \forall t \in[(m-1) L, m L-1],
\end{aligned}
$$

where $\mathbf{D}_{m}=\operatorname{diag}\left(\sigma_{m 1}^{2}, \sigma_{m 2}^{2}, \ldots, \sigma_{m K}^{2}\right)$ is the source covariance matrix at frame $m . \sigma_{m i}^{2}$ is the power of the $i$ th source and $\sigma_{n}^{2}$ is the power of noise. Vectorizing $\mathbf{R}_{m}$, we get the following $N^{2} \times 1$ vector:

$$
\mathbf{y}_{m}=\operatorname{vec}\left(\mathbf{R}_{m}\right)=\left(\mathbf{A}^{*} \odot \mathbf{A}\right) \mathbf{p}_{m}+\sigma_{n}^{2} \mathbf{1}_{m},
$$

where $\mathbf{p}_{m}=\left[\sigma_{m 1}^{2}, \sigma_{m 2}^{2}, \ldots, \sigma_{m K}^{2}\right]^{\mathrm{T}}$ and $\mathbf{1}_{m}=\left[\mathbf{e}_{1}^{\mathrm{T}}, \mathbf{e}_{2}^{\mathrm{T}}, \ldots, \mathbf{e}_{N}^{\mathrm{T}}\right]^{\mathrm{T}}$ with $\mathbf{e}_{1}^{\mathrm{T}}$ being a column vector of all zeros except a 1 at the $i$ th position. Comparing it with (2), we can say that $\mathbf{y}_{m}$ in (10) behaves like the data vector received at an array whose manifold is given by $\mathbf{A}^{*} \odot \mathbf{A}$, where $\odot$ denotes the Khatri-Rao (KR) product.

By stacking $\left[\mathbf{y}_{1}, \mathbf{y}_{2}, \ldots, \mathbf{y}_{M}\right] \triangleq \mathbf{Y}$, we have that

$$
\mathbf{Y}=\left(\mathbf{A}^{*} \odot \mathbf{A}\right) \mathbf{P}+\sigma_{n}^{2} \mathbf{E},
$$

where $\mathbf{P}=\left[\mathbf{p}_{1}, \mathbf{p}_{2}, \ldots, \mathbf{p}_{M}\right]$ and $\mathbf{E}=\left[\mathbf{1}_{1}, \mathbf{1}_{2}, \ldots, \mathbf{1}_{M}\right]$.

The noise subspaces $\mathbf{U}_{n}$ can be obtained by applying singular value decomposition (SVD) on $\mathbf{Y}$, which satisfies that

$$
\mathbf{U}_{n}^{H}\left(\mathbf{a}^{*}\left(\Omega_{i}\right) \otimes \mathbf{a}\left(\Omega_{i}\right)\right)=0, \quad i=1,2, \ldots, K .
$$


Then, the conventional subspace-based DOA estimation approach as MUSIC can be exploited as follows:

$$
\begin{aligned}
& P_{\text {music }}(\Omega) \\
& \quad=\frac{1}{\left(\mathbf{a}^{*}(\Omega) \otimes \mathbf{a}(\Omega)\right)^{H} \mathbf{U}_{n} \mathbf{U}_{n}^{H}\left(\mathbf{a}^{*}(\Omega) \otimes \mathbf{a}(\Omega)\right)} .
\end{aligned}
$$

It is interesting to note that spectrum peak search process is applied in the spatial frequency domain instead of the angle domain. The source signals with different frequencies and angles correspond to respective spatial frequency; therefore they can be separated in the spatial frequency domain. Since the spatial frequency includes both frequency and angle information, we will show how to determine their matching relation in the following part.

3.2. Pair Matching. According to the definition of spatial frequency in (5), once we know the frequency of each signal and match it to the corresponding spatial frequency, DOA can be obtained by simple computation. Hence, the pair matching of frequency and angle is equivalent to that of frequency and spatial frequency.

Consider the frequency domain model of (2), which is given by

$$
\mathbf{X}\left(f_{j}\right)=\mathbf{A}\left(f_{j}\right) \mathbf{S}\left(f_{j}\right)+\mathbf{N}\left(f_{j}\right), \quad j=1,2, \ldots, J,
$$

where $\mathbf{X}\left(f_{j}\right)$ is the received data at the array with frequency $f_{j}$, which is achieved by the Fourier transform of $\mathbf{x}(t)$. Our pair matching process is targeted at finding one or more spatial frequencies corresponding to $\mathbf{X}\left(f_{j}\right)$ among those that have been already estimated. We can treat this problem as a sparse recovery problem [12]; that is,

$$
\begin{array}{ll}
\operatorname{minimize} & \|\mathbf{v}\|_{0} \\
\text { subject to } & \mathbf{X}\left(f_{j}\right)=\mathbf{A}_{\text {Dict }} \mathbf{v},
\end{array}
$$

where

$$
\mathbf{A}_{\text {dict }}=\left[\mathbf{a}\left(\widehat{\Omega}_{1}\right), \mathbf{a}\left(\widehat{\Omega}_{2}\right), \ldots, \mathbf{a}\left(\widehat{\Omega}_{K}\right)\right]
$$

$\mathbf{A}_{\text {dict }}$ is an overcomplete dictionary composed of the steering vector generated by the estimated values of spatial frequency. $\mathbf{v}$ is the sparse vector whose position of nonzero elements represents the spatial frequency matched to $\mathbf{X}\left(f_{j}\right)$. However, the $l_{0}$-minimization is NP-hard in general. Thus, it is often converted into $l_{1}$-minimization [13] as follows:

$$
\begin{array}{ll}
\operatorname{minimize} & \|\mathbf{v}\|_{1} \\
\text { subject to } & \mathbf{X}\left(f_{j}\right)=\mathbf{A}_{\text {Dict }} \mathbf{v} \text {. }
\end{array}
$$

The support of $\mathbf{v}$ can be obtained by convention sparse recovery algorithms, such as Basis Pursuit (BP) [14] and Orthogonal Matching Pursuit (OMP) [15]. Considering the scenario that some of the source signals are with the same frequency, the sparse recovery problem in the case of high sparsity ratio may exist. Kwon et al. proposed a multipath matching pursuit (MMP) algorithm [16], which has been proved effective in high sparsity ratio case. Therefore, the MMP sparse recovery algorithm is selected.

As it is described above, it is possible to find the matched spatial frequency in the dictionary $\mathbf{A}_{\text {dict }}$ for each $\mathbf{X}\left(f_{j}\right)$ with the MMP algorithm.

3.3. Frequency and DOA Estimation. The set of $\left\{\widehat{f}_{j}\right\}_{j=1}^{J}$ achieved by Fourier transform on the rows of $\mathbf{x}(t)$ is regarded as the estimation of frequency. Since the pair matching for the set of $\left\{\widehat{f}_{j}\right\}_{j=1}^{J}$ and $\left\{\widehat{\Omega}_{i}\right\}_{i=1}^{K}$ has been already implemented, the DOA estimation is given by

$$
\widehat{\theta}_{i}=\arcsin \left(\frac{c \widehat{\Omega}_{i}}{\widehat{f}_{j}^{(i)}}\right),
$$

where $\widehat{f}_{j}^{(i)}$ denotes the frequency corresponding to $\widehat{\Omega}_{i}$. As $\widehat{\theta}_{i}$ is obtained by the matched $\widehat{f}_{j}$ and $\widehat{\Omega}_{i}, \widehat{\theta}_{i}$ and $\widehat{f}_{j}$ are pair matched automatically.

3.4. Performance Analysis. The implementation steps of the proposed method are summarized as follows.

Given: a received signal sequence $\{\mathbf{x}(t)\}_{t=0}^{T-1}, T=M L$, a source number $K$, and a frame length $L$.

Step 1. Compute $\mathbf{R}_{m}, \mathbf{y}_{m}=\operatorname{vec}\left(\mathbf{R}_{m}\right)$, for $m=1,2, \ldots, M$. Then, form a data matrix $\mathbf{Y}=\left[\mathbf{y}_{1}, \mathbf{y}_{2}, \ldots, \mathbf{y}_{M}\right]$.

Step 2. Perform SVD on $\mathbf{Y}$ in order to obtain the noise subspace matrix $\mathbf{U}_{n}$. Then, compute the spatial frequency spectrum by (13) and obtain $\widehat{\Omega}_{i}$, for $i=1,2, \ldots, K$.

Step 3. Compute the Fourier transform of $\mathbf{x}(t)$ and pick up the data $\mathbf{X}\left(f_{j}\right)$ for each frequency $f_{j}$, for $j=1,2, \ldots, J$.

Step 4. Compute the support of $\mathbf{X}\left(f_{j}\right)$ in $\mathbf{A}_{\text {Dict }}$ by sparse recovery and determine the corresponding $\widehat{\Omega}_{i}$ to $\widehat{f}_{j}$.

Step 5. Compute $\theta_{i}$ by (18) with the matched $\widehat{\Omega}_{i}$ and $\widehat{f}_{j}$.

Step 6. Repeat Steps 4 and 5 for each $\mathbf{X}\left(f_{j}\right)$ and obtain the set $\left\{\widehat{\theta}_{i}\right\}_{i=1}^{K}$ as the DOA estimation.

It is obvious that the performance of DOA estimation is related to the spatial frequency and frequency. The precision of frequency estimation is due to discrete Fourier transform (DFT) points $N_{L}$, whose maximum value is the number of snapshot. From (18), we have

$$
\frac{d \theta}{d f}=\frac{\Omega c}{f^{2} \sqrt{1-\Omega^{2} c^{2} / f^{2}}} .
$$

This means that the influence of frequency estimation error on the DOA estimation decreases with the growth of frequency. Therefore, the DOA estimation precision is mainly 
due to the spatial frequency estimation precision when the carrier frequency is high. As the nested array, which is capable of extending the array aperture effectively, is utilized in spatial frequency estimation, we can achieve high estimation precision compared to the ULA. If the spatial frequency is estimated accurately, the DOA estimation with high precision follows.

In fact, the broadband DOA estimation problem includes two-dimensional parameter: frequency and angle. It is normally implemented with a two-dimensional search process in frequency and angle domain. Compared with traditional method, the proposed method only needs to conduct spectral searching process once in the spatial frequency domain and a simple pair matching process. However, the traditional method, which uses band-pass filters for each element and obtains narrowband DOA estimation by MUSIC, needs multiple search processes for the signals with different frequencies. Therefore, the proposed method reduces the computational complexity to some extent.

\section{Simulation Results}

In this section, we provide several sets of simulation results to demonstrate the performance of the proposed method. In all the simulation examples below, we suppose that $f \in$ $(0.1,1.5) \mathrm{GHz}, \theta \in\left(-90^{\circ}, 90^{\circ}\right)$, for all the source signals. The root mean square error (RMSE) of angle from $L$ Monte Carlo trials is used as our performance measure and it is defined as

$$
\text { RMSE }=\sqrt{\frac{1}{K L} \sum_{i=1}^{K}\left|\theta_{i}-\hat{\theta}_{i}\right|^{2}},
$$

where $\theta_{i}$ and $\hat{\theta}_{i}$ denote the true and estimation DOAs, respectively.

\subsection{Simulation and Setting}

Example 1. This simulation example considers an underdetermined case where 13 uncorrelated source signals $(K=$ 13) impinge on the nested array with 6 sensors $\left(N_{1}=N_{2}=\right.$ $3)$. The number of snapshots $T(T=M L)$ and SNR are set to $M \times L(M=32, L=1024)$ and $15 \mathrm{~dB}$, respectively. The true DOAs are given by $\left\{\theta_{1}, \theta_{2}, \ldots, \theta_{K}\right\}=\left\{-60^{\circ},-51^{\circ},-40^{\circ}\right.$, $\left.-30^{\circ},-20^{\circ},-7^{\circ}, 5^{\circ}, 18^{\circ}, 25^{\circ}, 31^{\circ}, 45^{\circ}, 53^{\circ}, 60^{\circ}\right\}$ with the carrier frequency $\left\{f_{1}, f_{2}, \ldots, f_{K}\right\}=\{1.15,1.03,1.03,0.57,1.35,0.8$, $1.03,1.35,0.57,1.35,0.8,1.03,1.15 \mathrm{GHz}$. Figures 2 and 3 show the spatial frequency and frequency estimated results, respectively, and Figure 4 shows the pair matching results of frequency and angle by MMP algorithm.

Example 2. In this simulation example, we consider the spatial frequency estimation performance with respect to SNR. Five source signal angles and frequencies are set to $\{1.0,0.9,1.1,1.2,1.3\} \mathrm{GHz}$ and $\left\{-30^{\circ},-15^{\circ}, 5^{\circ}, 15^{\circ}, 30^{\circ}\right\}$, respectively. The SNR varies from $-10 \mathrm{~dB}$ to $15 \mathrm{~dB}$ in $2.5 \mathrm{~dB}$ intervals. Figure 5 shows the RMSE of the spatial frequency estimation as a function of SNR, averaged over 1000 Monte Carlo trials.

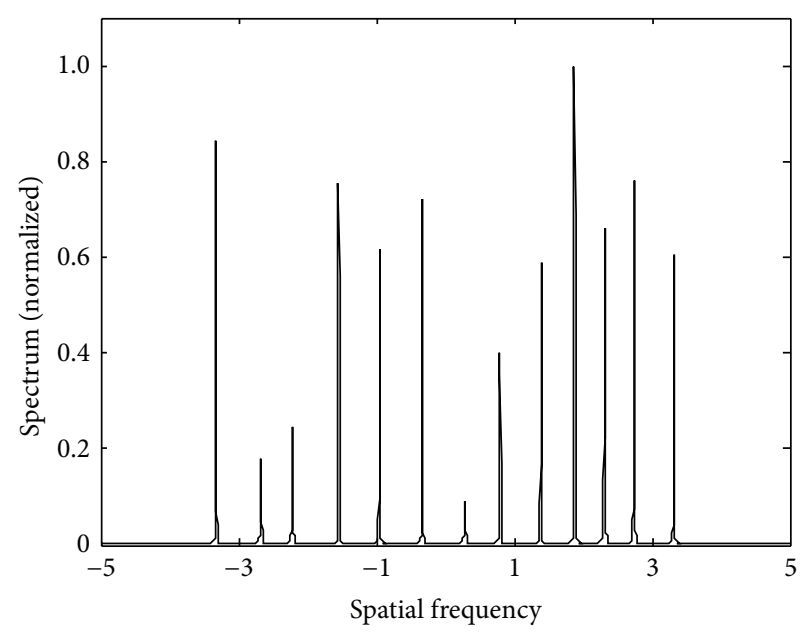

FIgURE 2: Spatial frequency estimated results of 13 source signals, $N=6, M=32, L=1024$, and $\mathrm{SNR}=15 \mathrm{~dB}$.

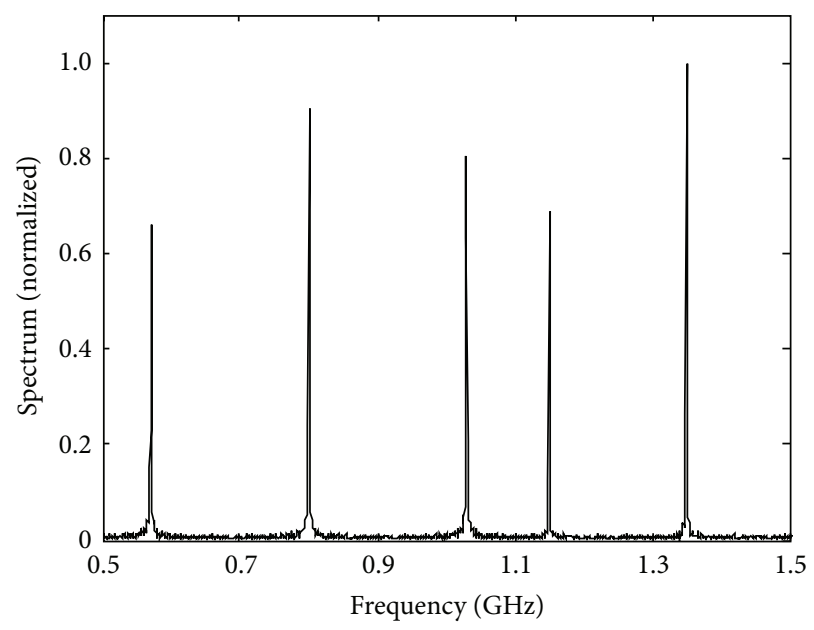

FIgURE 3: Frequency estimated results, $N_{L}=4096$ and $\mathrm{SNR}=15 \mathrm{~dB}$.

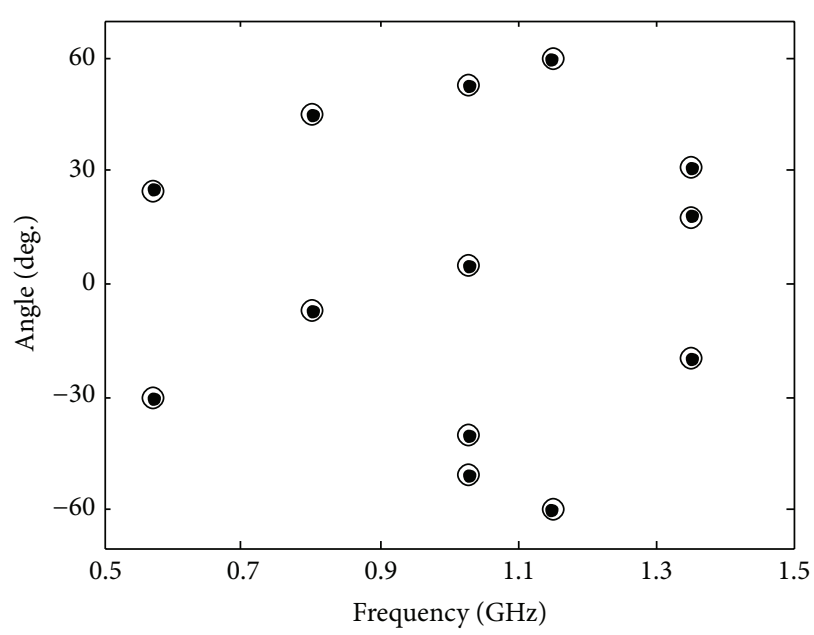

Figure 4: Pair mathcing results of 13 source signals, $\mathrm{SNR}=15 \mathrm{~dB}$. 


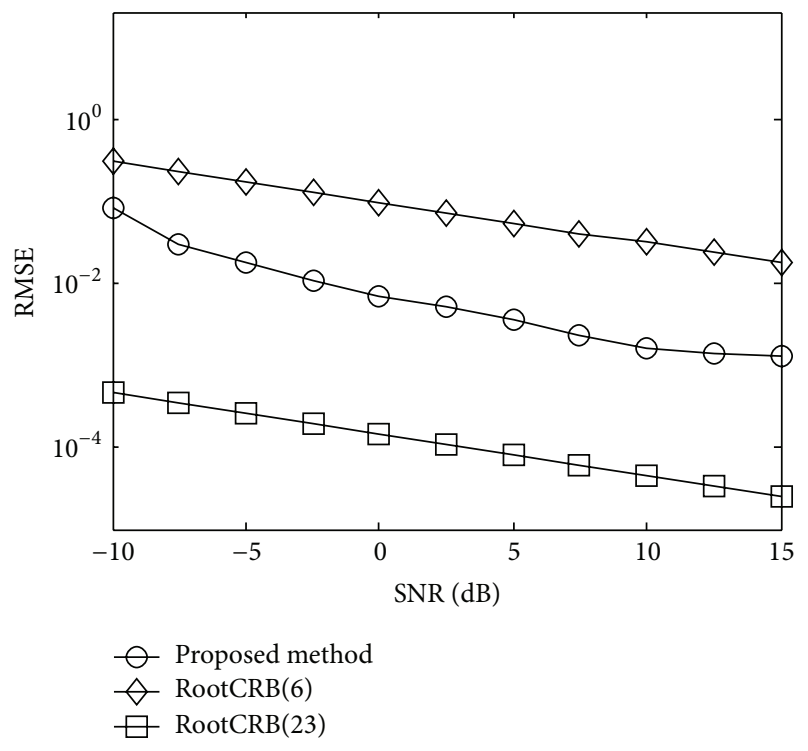

FIGURE 5: RMSE of spatial frequency versus SNR, $N=6$ and $K=5$.

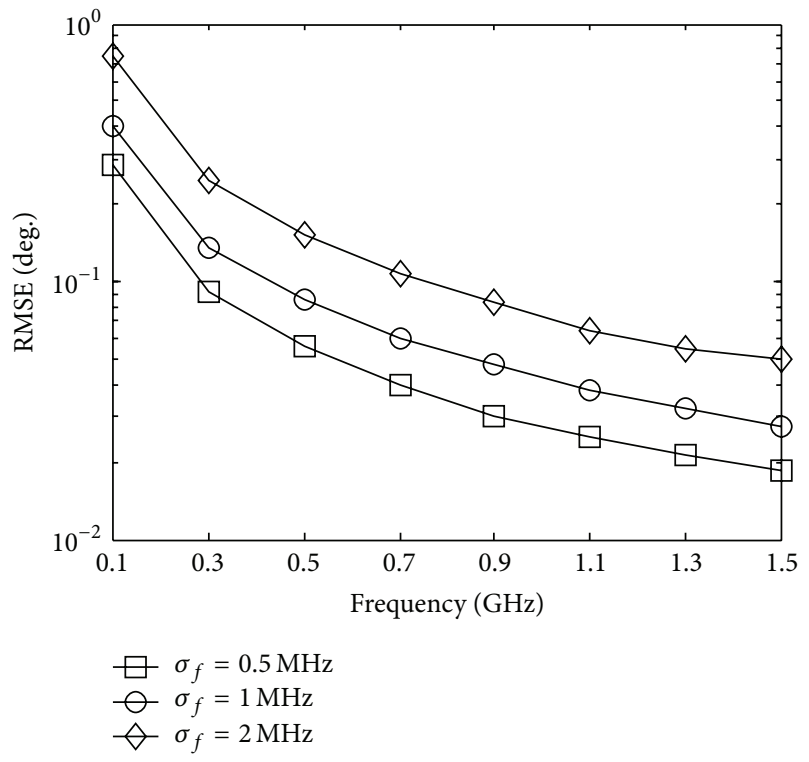

FIGURE 6: RMSE of angle versus frequencies, $N=6, K=3$, and $\mathrm{SNR}=15 \mathrm{~dB}$.

Example 3. In this simulation example, we consider the influence of frequency estimated error on RMSE of angle. Three DOAs are set to $-15^{\circ}, 30^{\circ}$, and $45^{\circ}$ with $\mathrm{SNR}=15 \mathrm{~dB}$. The frequency varies from $0.1 \mathrm{GHz}$ to $1.5 \mathrm{GHz}$ with interval $0.2 \mathrm{GHz}$. Every fixed frequency conducts 1000 Monte Carlo trials. Figure 6 shows the RMSE of angles as a function of frequency with different $\sigma_{f}$ (standard deviation of frequency estimation).

Example 4. In this example, we examine the DOA estimation performance with respect to SNR and snapshots. The simulation conditions are similar to those of Example 1 except

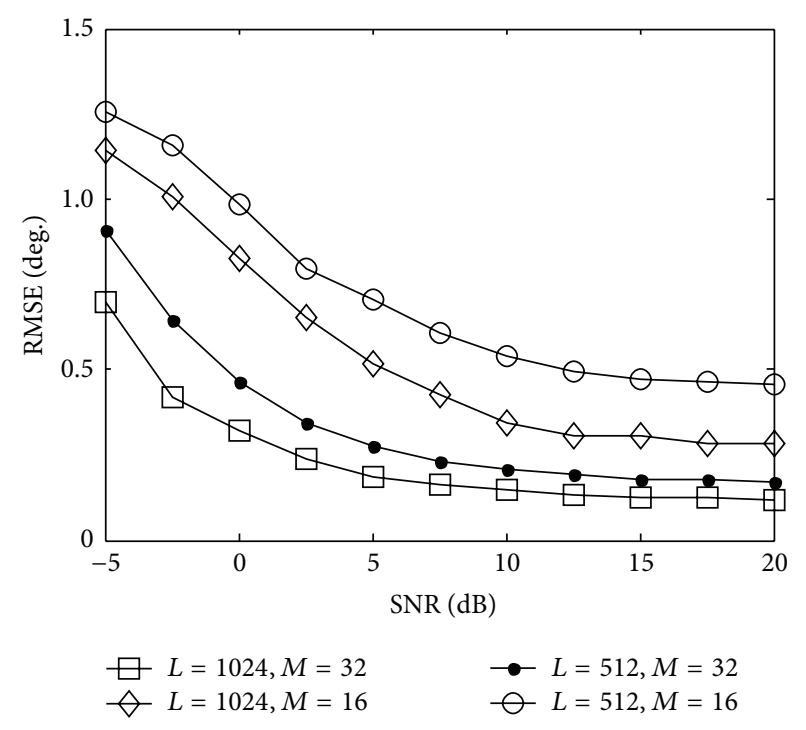

FIgURE 7: RMSE of angle versus SNR and snapshots, $N=6$ and $K=13$.

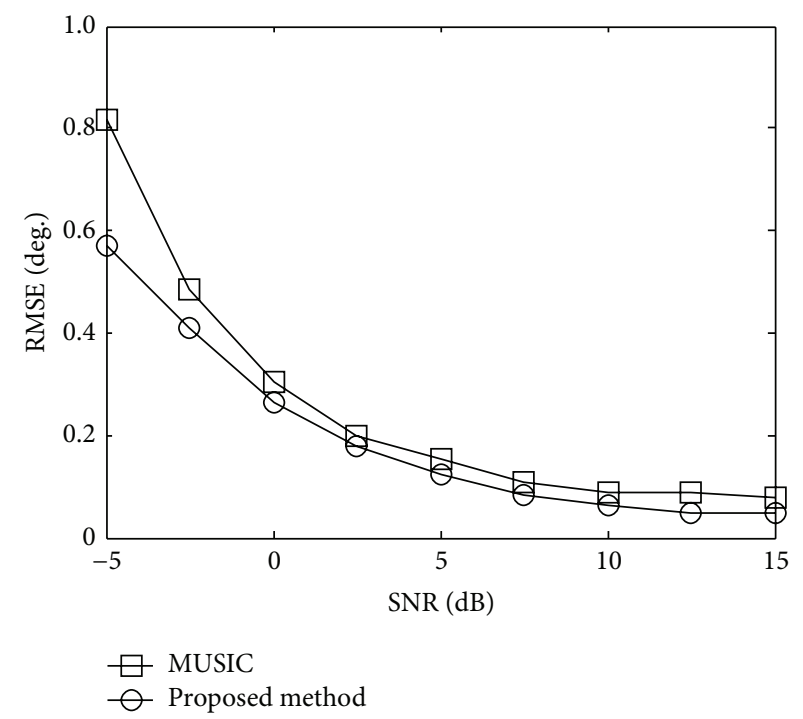

FIGURE 8: RMSE comparison, $N=4, K=5$, and SNR $=15 \mathrm{~dB}$.

the SNR and snapshots. The SNR varies from $-5 \mathrm{~dB}$ to $20 \mathrm{~dB}$ in $2.5 \mathrm{~dB}$ intervals. The RMSE of angle as a function of SNR with different snapshots is shown in Figure 7.

Example 5. In this example, we compare with traditional method, which uses band-pass filters for each element and obtains narrowband DOA estimation by MUSIC. The SNR varies from $-5 \mathrm{~dB}$ to $15 \mathrm{~dB}$ in $2.5 \mathrm{~dB}$ intervals. We choose five source signals with different frequencies and DOAs. Every fixed SNR conducts 1000 Monte Carlo trials. Figure 8 compares the RMSE of the proposed method and MUSIC method based on band-pass filtering. The CPU runtime of MUSIC and the proposed method as a function of source number are shown in Figure 9. 


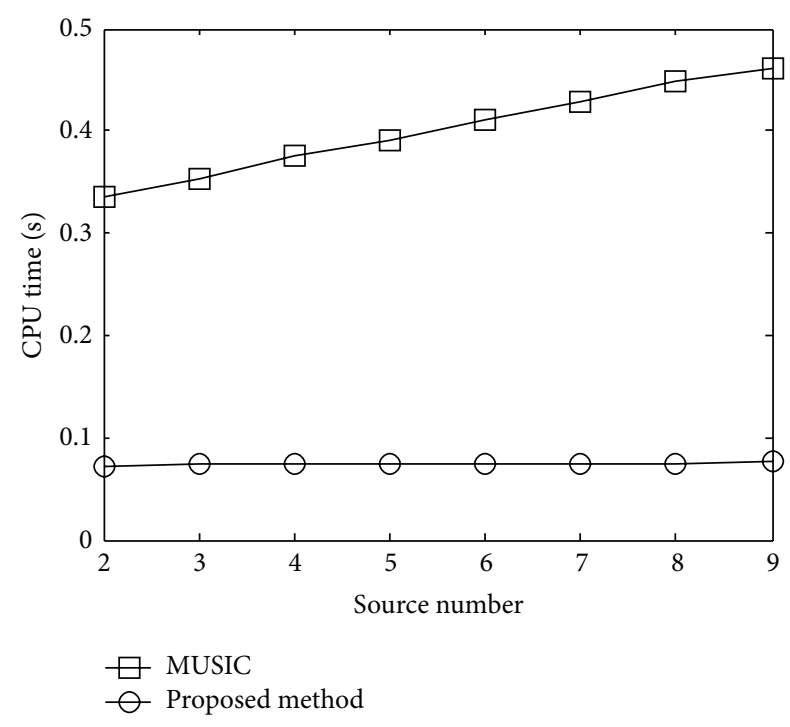

FIGURE 9: CPU time comparison, $N=6, L=1024$, and $M=32$.

4.2. Discussion and Analysis. From Figures 2 and 3, we see that the proposed method is effective for spatial frequency and frequency estimation. In Figure 4, $\bigcirc$ and $\bigcirc$ denote the true values and estimated values, respectively, which prove that the frequency and angle can be pair matched correctly by MMP algorithm.

The $\operatorname{RootCRB}(N)$ in Figure 5 denotes the square root of Cramer-Rao bound with $N$ sensors. It is interesting to note that the RootCRB is for spatial frequency instead of angle. Since the nested array increases the DOF from $N$ to $\left(N^{2}-\right.$ $2) / 2+N$ ( $N$ is even) [4], we compare it with the $\operatorname{RootCRB}(6)$ and RootCRB(23). From Figure 5, we observe that the nested array with 6 sensors yields better RMSE of spatial frequency than the ULA with 6 sensors, but still not as good as the ULA with 23 sensors.

The results of Figure 6 show that the smaller $\sigma_{f}$ is, the better RMSE we can obtain. However, with the same $\sigma_{f}$, the RMSE of angle will decrease when the frequency varies from $0.1 \mathrm{GHz}$ to $1.5 \mathrm{GHz}$. Therefore, we can conclude that the frequency estimation error has less influence at higher frequencies, and vice versa for lower frequencies, which is equal to the analysis in (19).

From Figure 7, we see that better RMSE of angle can be obtained by increasing $M$ with fixed $L$ or increasing $L$ with fixed $M$, as it increases the number of snapshots $T=M L$ in both scenarios. However, when the number of snapshots is fixed at $T=16384$, the case of $L=512$ and $M=32$ provides better RMSE than that of $L=1024$ and $M=16$. Hence, this set of empirical results suggests that, for a fixed $T$, trying to obtain more frames $M$ by decreasing the frame length $L$ ( $L$ should not be overly small) tends to be a better option for performance improvement than the opposite.

From Figure 8, we see that the RMSE of the proposed method is better than MUSIC. This is because it extends the array aperture by nested array, which improves the DOA estimation precision. The results of Figure 9 show that CPU time of the proposed method is less than MUSIC and varies little with the growth of source number. The reason is that the proposed method can estimate the spatial frequencies of all the signals in a single spectral search process, which is less affected by source number. However, the times of spectral search in MUSIC are related to the source number, which equals the number of signals with different frequencies. Therefore, the CPU runtime becomes longer significantly when the source number is large.

\section{Conclusion}

This paper has addressed the broadband DOA estimation problem with nested array. The proposed method can obtain the frequency and angle estimation of multiple source signals by spatial frequency estimation and pair matching with sparse recovery. The pair matching process is conducted in the set of spatial frequency that have been estimated for reducing the computation. Moreover, the proposed method can work in broadband, underdetermined, and low SNR cases, which is very suitable for electronic reconnaissance system.

\section{Conflict of Interests}

The authors declare that there is no conflict of interests regarding the publication of this paper.

\section{Acknowledgments}

This work is partially supported by the National Basic Research Program under Grant no. 61XX81, National Defense Pre-Research Program during the "12th Five-Year Plan" under Grant no. X110XX2030, and Fundamental Research Funds for the Central Universities of China under Grant no. K5051202026.

\section{References}

[1] N. Yu, X. H. Qi, and X. L. Qiao, "Multi-channels wideband digital reconnaissance receiver based on compressed sensing," IET Signal Processing, vol. 7, no. 8, pp. 731-742, 2013.

[2] Z. Liu, X. Wang, G. Zhao, G. Shi, and J. Lin, "Wideband DOA estimation based on sparse representation," in Proceedings of the IEEE International Conference on Signal Processing, Communications and Computing, pp. 1-4, Yunnan, China, August 2013.

[3] D. S. Talagala, W. Zhang, and T. D. Abhayapala, "Broadband DOA estimation using sensor arrays on complex-shaped rigid bodies," IEEE Transactions on Audio, Speech and Language Processing, vol. 21, no. 8, pp. 1573-1585, 2013.

[4] M. Agrawal and S. Prasad, "Broadband DOA estimation using 'spatial-only' modeling of array data," IEEE Transactions on Signal Processing, vol. 48, no. 3, pp. 663-670, 2000.

[5] P. Pal and P. P. Vaidyanathan, "Nested arrays: a novel approach to array processing with enhanced degrees of freedom," IEEE Transactions on Signal Processing, vol. 58, no. 8, pp. 4167-4181, 2010.

[6] P. Pal and P. P. Vaidyanathan, "Multiple level nested array: an efficient geometry for $2 q$ th order cumulant based array 
processing," IEEE Transactions on Signal Processing, vol. 60, no. 3, pp. 1253-1269, 2012.

[7] S. Li, W. He, X. Yang, M. Bao, and Y. Wang, "Direction-of-arrival estimation of quasi-stationary signals using two-level KhatriRao subspace and four-level nested array," Journal of Central South University, vol. 21, no. 7, pp. 2743-2750, 2014.

[8] R. O. Schmidt, "Multiple emitter location and signal parameter estimation," IEEE Transactions on Antennas and Propagation, vol. 34, no. 3, pp. 276-280, 1986.

[9] J.-F. Gu, W.-P. Zhu, and M. N. S. Swamy, "Compressed sensing for DOA estimation with fewer receivers than sensors," in Proceedings of the IEEE International Symposium of Circuits and Systems (ISCAS '11), pp. 1752-1755, May 2011.

[10] K. Han and A. Nehorai, "Nested vector-sensor array processing via tensor modeling," IEEE Transactions on Signal Processing, vol. 62, no. 10, pp. 2542-2553, 2014.

[11] W.-K. Ma, T.-H. Hsieh, and C.-Y. Chi, "DOA estimation of quasi-stationary signals with less sensors than sources and unknown spatial noise covariance: a Khatri-Rao subspace approach," IEEE Transactions on Signal Processing, vol. 58, no. 4, pp. 2168-2180, 2010.

[12] E. J. Candes and T. Tao, "Near-optimal signal recovery from random projections: universal encoding strategies?" IEEE Transactions on Information Theory, vol. 52, no. 12, pp. 5406-5425, 2006.

[13] E. J. Candès, J. Romberg, and T. Tao, "Robust uncertainty principles: exact signal reconstruction from highly incomplete frequency information," IEEE Transactions on Information Theory, vol. 52, no. 2, pp. 489-509, 2006.

[14] S. S. Chen, D. L. Donoho, and M. A. Saunders, "Atomic decomposition by basis pursuit," SIAM Journal on Scientific Computing, vol. 20, no. 1, pp. 33-61, 1998.

[15] J. A. Tropp and A. C. Gilbert, "Signal recovery from random measurements via orthogonal matching pursuit," IEEE Transactions on Information Theory, vol. 53, no. 12, pp. 4655-4666, 2007.

[16] S. Kwon, J. Wang, and B. Shim, "Multipath matching pursuit," IEEE Transactions on Information Theory, vol. 60, no. 5, pp. 2986-3001, 2014. 

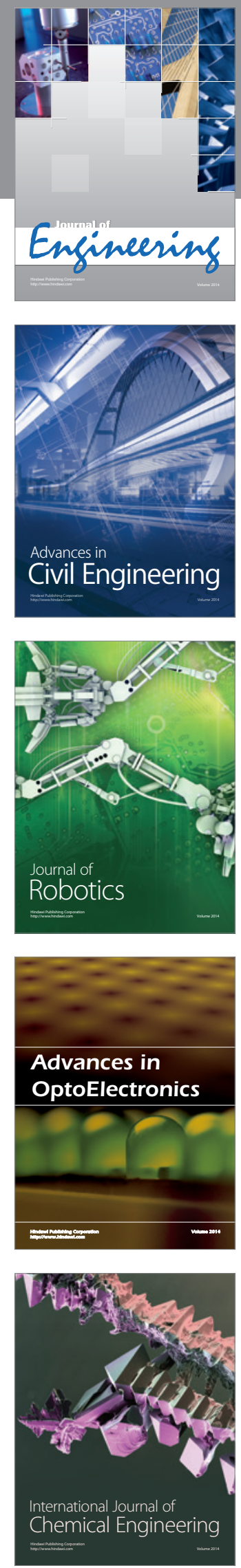

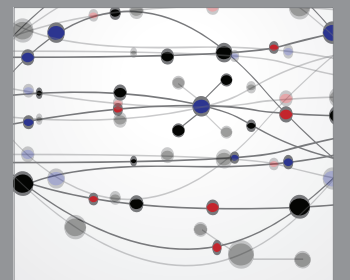

The Scientific World Journal
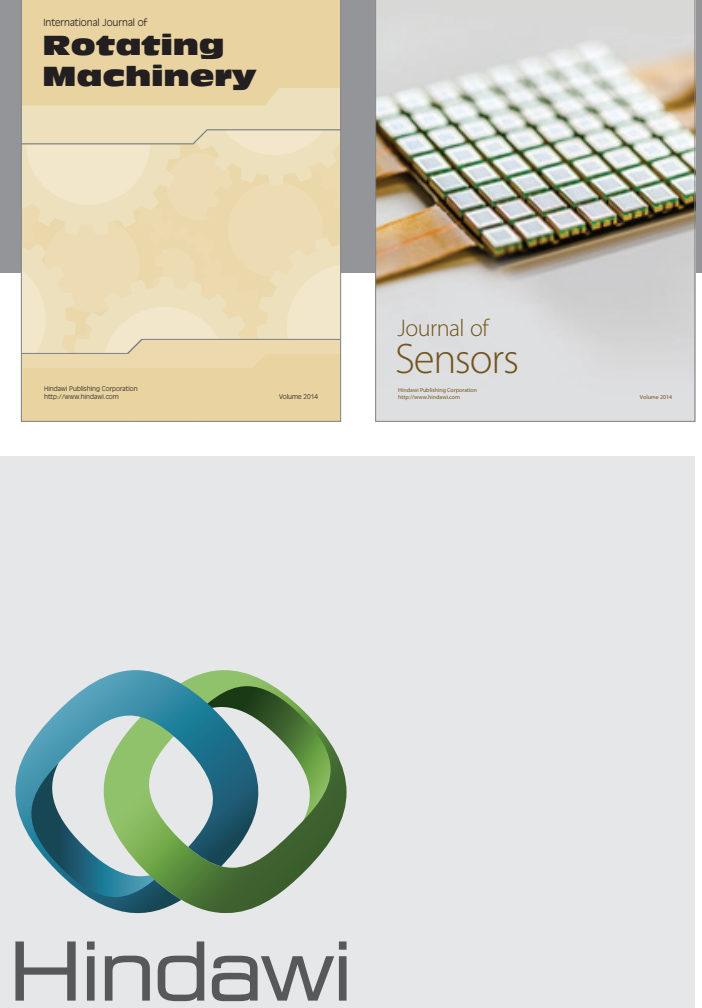

Submit your manuscripts at http://www.hindawi.com
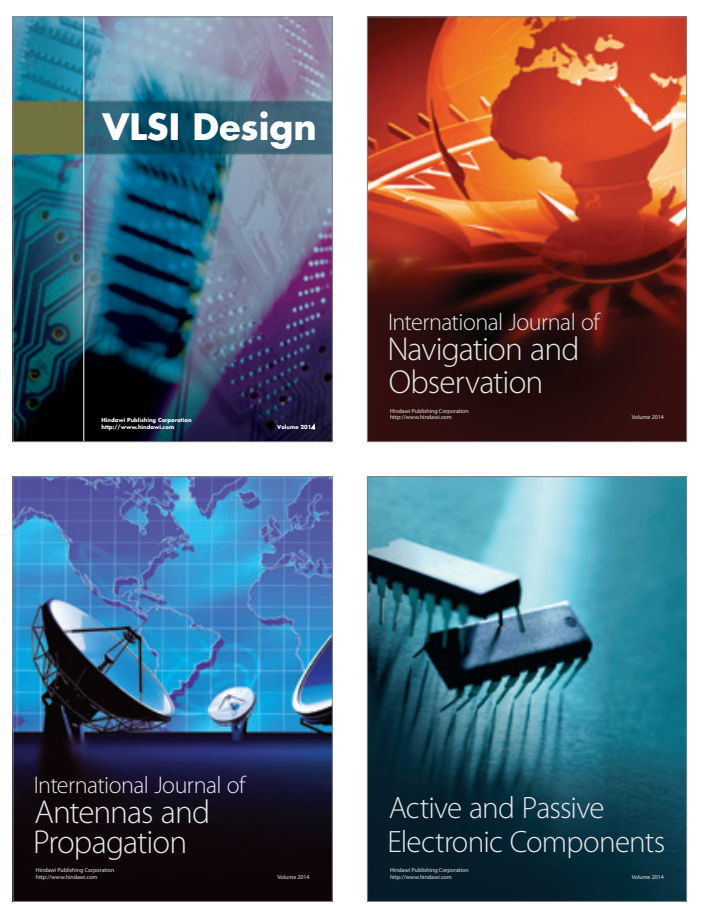
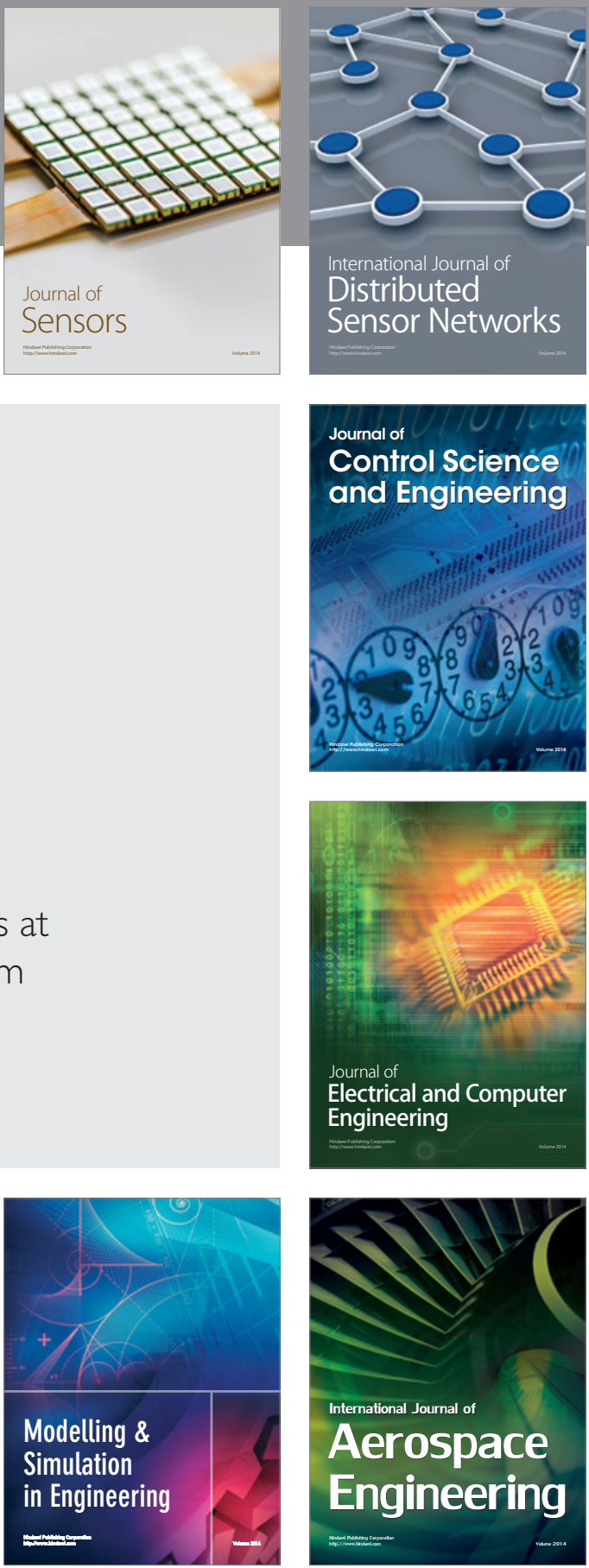

Journal of

Control Science

and Engineering
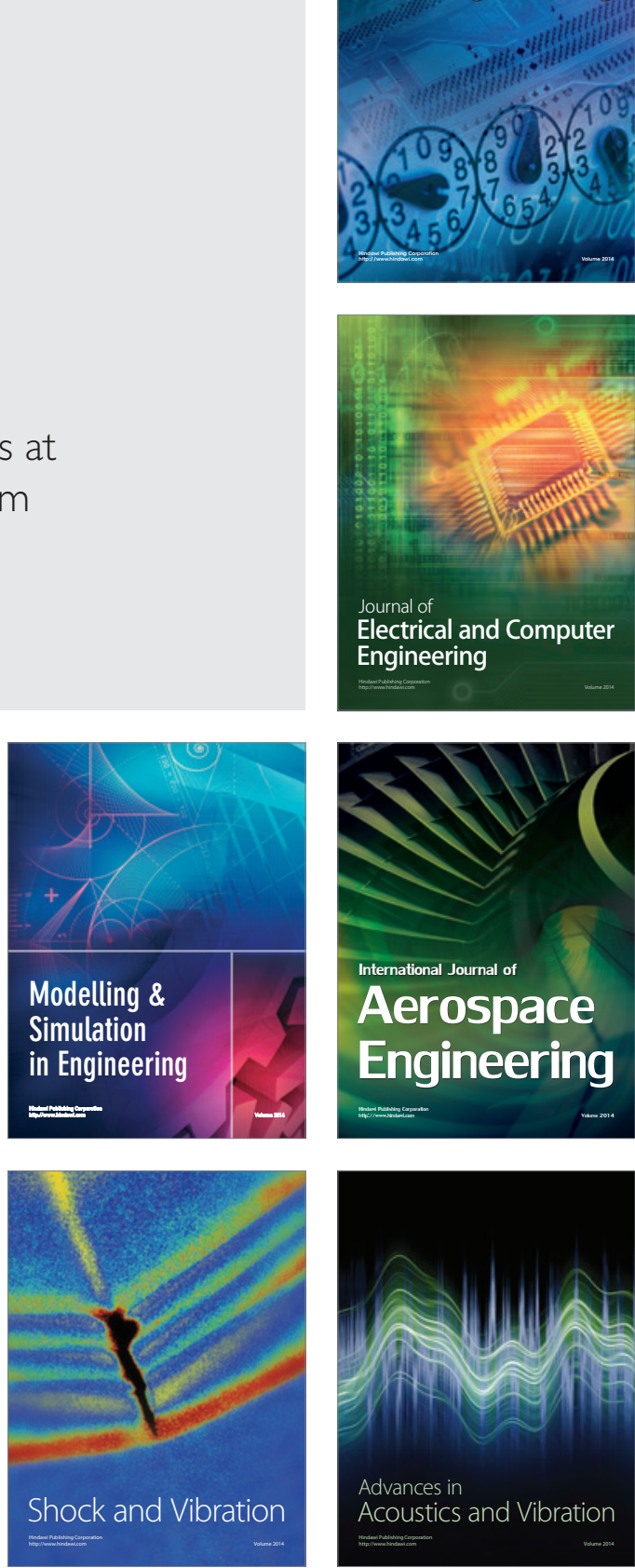